\title{
Multisite Generalization of the SHArP Weather Generator
}

\author{
KIMBERLy SMITH AND COURTENAY STRONG \\ Department of Atmospheric Sciences, University of Utah, Salt Lake City, Utah \\ FIRAS RASSOUL-AGHA \\ Department of Mathematics, University of Utah, Salt Lake City, Utah
}

(Manuscript received 12 August 2017, in final form 25 June 2018)

\begin{abstract}
Generalization of point-scale stochastic weather generators to simultaneously produce output at multiple sites provides more powerful support for hydrology and climate change impact studies. Generalization preserves the statistical properties of each individual site while maintaining proper spatial correlation over the domain. Here, generalization of the daily precipitation and temperature components of the stochastic harmonic autoregressive parametric (SHArP) weather generator is presented. The generalization process for temperature involves conversion of vector time series to matrix time series that capture between-site covariances of maximum and minimum daily temperature. Between-site temperature covariances depend on spatial precipitation-occurrence patterns (POPs), of which there are up to $2^{M}$ for $M$ sites. To dramatically reduce the number of covariance matrices that drive temperature, multisite SHArP uses empirical orthogonal function analysis to categorize the POPs and harmonic smoothing to reduce the number of parameters describing the temporal evolution (annual cycle) of the elements in the covariance matrices. By modeling precipitation-regime-specific residuals, the model is shown to capture statistically significant spatial and temporal contrasts in observed temperature covariance. For precipitation simulation, we extend existing techniques by adding a trend term to the occurrence and amount parameters. Multisite generalization of the framework is illustrated by simulating stochastic historical and future temperature and precipitation across complex terrain over northern Utah on the basis of historical station observations and historical and future statistically downscaled climate model output.
\end{abstract}

\section{Introduction}

Stochastic weather generators (SWGs) were primarily introduced to simulate daily meteorological variables, namely precipitation and temperature, that replicate statistical properties of the observed data at the location in question. SWGs are especially useful tools for hydrologists, climate scientists, agriculturalists, ecologists, planners, engineers, and practitioners in related fields given missing meteorological data or an interest in ensemble statistics (e.g., for uncertainty analysis). The development of SWGs often begins with the precipitation process since most other meteorological variables depend on whether or not precipitation occurred, and the addition of air temperature is a natural next step. SWGs are constructed to work on a point scale, but to further capture variations

\footnotetext{
Corresponding author: Courtenay Strong, court.strong@utah. edu
}

between sites or examine hydrologic or climate change impacts on a broader scale the methods need to be generalized to multiple sites. Generalization to multiple sites has its own set of challenges, especially as the number of sites increases.

Wilks (1998) introduced the widely known multisite generalization model of precipitation occurrence and amount based on chain-dependent processes (a two-state, second-order Markov chain for occurrence and a mixed exponential distribution for amount) that were described in Todorovic and Woolhiser (1975) and later applied in Richardson (1981). This is done by applying spatially correlated yet time-independent random vectors on the models of each individual site within the domain (Wilks 1998). With this method, each site retains its own statistical properties while maintaining realistic correlations with the neighboring sites.

Wilks (1999b) expanded on the multisite generalization method presented in Wilks (1998) by applying the 
method over an area with complex terrain in the western United States. In addition, the method was expanded to include daily maximum and minimum temperature and solar radiation following Richardson (1981). The Richardson (1981) method involves simulating the residuals of air temperature and solar radiation. Fitted correlation functions were used to capture the seasonal variations in the study area, and this multisite generation was able to model the precipitation over complex terrain while preserving the spatial correlations found in nature (Wilks 1999b). Later, Wilks (2009) showed the practicality of a spatially coherent SWG that interpolated parameters for single sites as described in Wilks (2008). In addition, the study was able to synchronize the gridded synthetic data to true weather data at reference stations within the domain and provide more realistic simulations for hydrologic purposes.

Caraway et al. (2014) developed a nonparametric multisite SWG using the $k$-nearest neighbor resampling approach. This model uses clustering of homogeneous sites in addition to Markov chain states to simulate precipitation at multiple sites within a heterogeneous watershed. While most present-day weather generators are parametric and are based off the work of Richardson (1981) and Wilks (1998), including the stochastic harmonic autoregressive parametric weather generator (SHArP; Smith et al. 2017) and Multi-site Weather Generator of École de Technologie Supérieure (MulGETS; Chen et al. 2014), the advantages of a nonparametric weather generator include the ability to capture the nonlinear variability that is missed in the linear parametric SWGs. Kleiber et al. (2012) introduced a generalized linear model (GLM) that uses spatial Gaussian processes to model the statistical parameters of precipitation over a domain. A similar nonparametric GLM for maximum and minimum temperature was also developed and is described in Kleiber et al. (2013). Verdin et al. (2015) combined the methods in Kleiber et al. (2012) and Kleiber et al. (2013) and developed a GLM-based weather generator that can be applied to any area regardless of data density or availability.

Existing multisite SWG research has focused primarily on the precipitation component of the generator and the complexities associated with generating it realistically at multiple sites. There is comparatively minimal focus on the temperature component, but temperature fidelity is important for determining the phase (snow vs rain) and fate of winter precipitation over complex terrain. Many previous studies involving the temperature component have opted to use the method applied in Richardson (1981), which was generalized to multiple sites over complex terrain in Wilks (1999b). This method involves prescribing and then removing the means and standard deviations of the temperature values (separating the dry and wet days) in advance and generating the temperature residuals. The resulting mean temperatures switch abruptly between the dry- and wet-state values due to the prescribed means. To overcome this limitation and provide a more realistic temporal evolution of daily weather, Smith et al. (2017) introduced the SHArP weather generator, which simulates autocorrelated temperatures directly without prescribing mean values in advance. The extension of existing multisite SWGs usually involves a conditioning of temperature on precipitation occurrence, but this is largely sitewise, meaning variations in the larger-scale spatial pattern of precipitation do not affect the temporal evolution or spatial covariance of temperature.

In this study, we present a multisite generalization of SHArP. The mathematical formulation follows that of the single-site, single-temperature case in Smith et al. (2017), but major differences stem from how objectively identified precipitation-occurrence patterns (POPs) impact temperature stochasticity and autocorrelation. In the single-site, single-temperature case, we used a temporally varying noise coefficient that depended on whether the given day was wet or dry at the site. Having multiple sites introduces between-site covariances that are found to depend on POPs whose number increases as $2^{M}$ for $M$ sites. To circumvent this situation, we use empirical orthogonal function analysis to objectively categorize the POPs, yielding a compact set of matrices for driving between-site temperature covariance. An example application in the western United States is used to illustrate the fidelity of the framework in complex terrain where precipitation patterns can change markedly over the study domain, and the simulation period is 1950-2100 to illustrate how trends are handled.

\section{Data and study area}

SHArP was initially developed, tested, and presented in an entirely observational context (Smith et al. 2017). We use observations again here and incorporate statistically downscaled historical and future climate model output in part because the principal anticipated application of SHArP is simulation of future weather under climate change. From the Global Historical Climatology Network (Menne et al. 2012), we use daily precipitation, minimum temperature, and maximum temperature from 19 sites over northern Utah and southwestern Wyoming (Fig. 1) during 1950-2005. Additional available stations that had $10 \%$ or more missing values over this period were eliminated.

We also use $0.125^{\circ}$ bias-corrected constructed analogs (BCCA) of daily CCSM4 output from phase 5 of the 


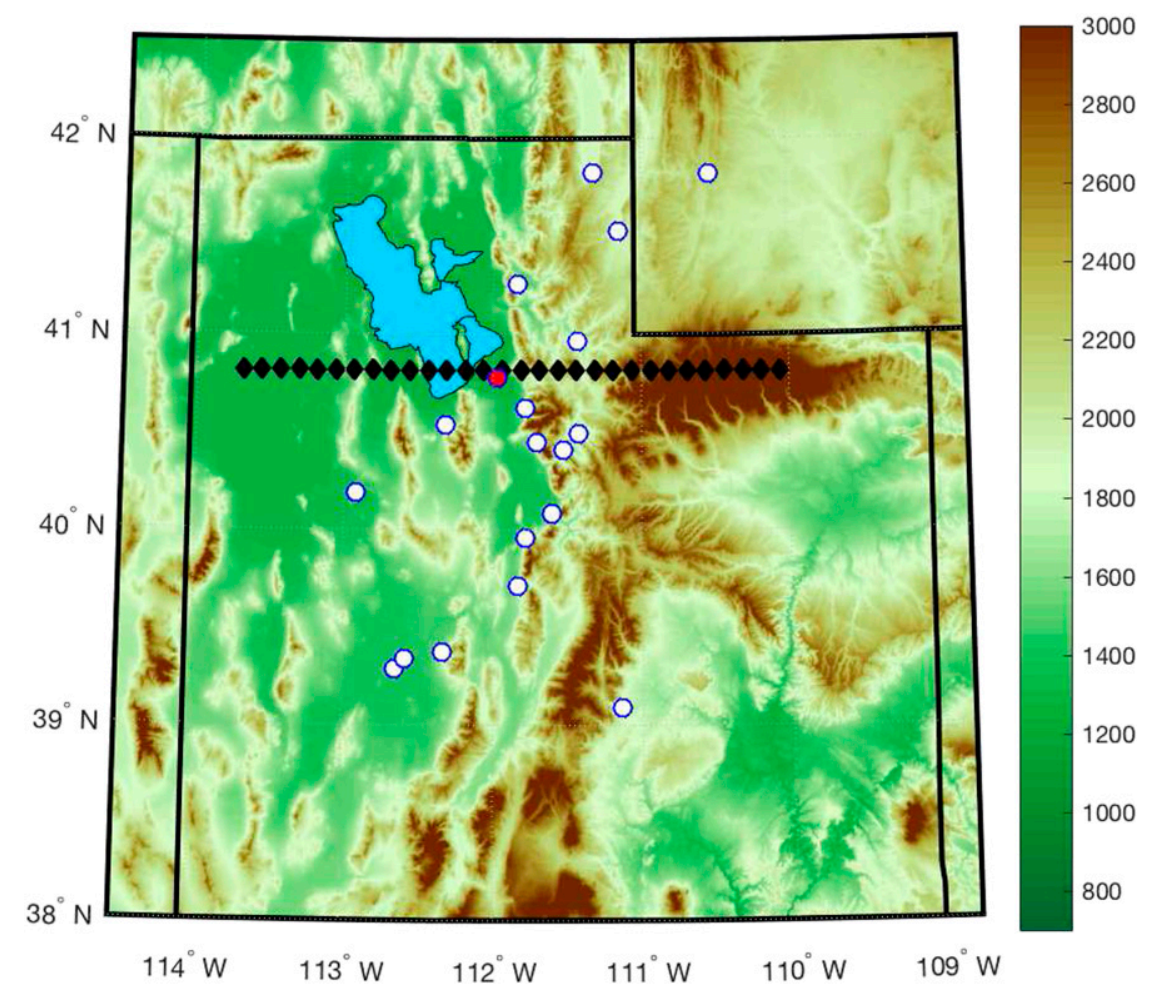

FIG. 1. Domain map showing northern and central Utah with locations of the 19 observation stations (circles, shaded red or white) and a transect of 30 grid points at which statistically downscaled climate model output was used (diamonds). The transect was chosen to be the row of grid points closest to KSLC (indicated by the red circle). Color shading indicates elevation (m MSL), with the Great Salt Lake shown in cyan.

Coupled Model Intercomparison Project (CMIP5; Maurer et al. 2007; Bureau of Reclamation 2013). We used the historical BCCA data, which span from 1 January 1950 to 31 December 2005, in this analysis, as well as the future RCP 8.5 (high emissions scenario) data, which span from 1 January 2006 to 31 December 2100. We select from the statistically downscaled data a transect of 30 sites from the western desert of Utah $\left(40.8125^{\circ} \mathrm{N}, 113.6875^{\circ} \mathrm{W}\right)$ to the Uinta Mountains $\left(40.8125^{\circ} \mathrm{N}, 110.0625^{\circ} \mathrm{W}\right)$, which includes a point near the Salt Lake International Airport (KSLC; $40.8125^{\circ} \mathrm{N}$, $111.9375^{\circ} \mathrm{W}$; site 15 adjacent to the red circle in Fig. 1). Half of the sites are located in the "valley," and the other half of the sites are located in the mountains. The study region is located within the larger Great Basin, which is known for its semiarid climate and basin-andrange topography (e.g., Thompson and Burke 1974).

To pair with the statistically downscaled climate data (1950-2100), we use indices of El Niño-Southern Oscillation (ENSO; e.g., Diaz et al. 2001) and the Pacific decadal oscillation (PDO; e.g., McCabe and Dettinger 2002). These data are bandpass-filtered, spatially averaged historical CCSM4 sea surface temperature output processed by following the method of Smith et al. (2015).

\section{Multisite simulation of daily maximum and minimum air temperature}

\section{a. Model formulation}

The linear model for simulating multiple temperatures at multiple sites extends the SHArP linear model introduced in Smith et al. (2017) and is given by

$$
\mathbf{T}_{k+1}=\mathbf{A} \mathbf{T}_{k}+\mathbf{B}_{k}+\mathbf{C}_{k} \boldsymbol{\epsilon}_{k},
$$

where $\mathbf{A}$ is a $2 M \times 2 M$ autocorrelation matrix for number of sites $M, \mathbf{B}_{k}$ is a $2 M \times 1$ column vector that depends on day $k, \mathbf{C}_{k}$ is a $2 M \times 2 M$ positive definite matrix made up of noise coefficients, and $\boldsymbol{\epsilon}_{k}$ is a $2 M \times 1$ column vector. Errors $\boldsymbol{\epsilon}_{k}$ are independent and identically distributed (i.i.d.) random vectors with entries that themselves are independent standard normals. The temperature on day $k+1$ is dependent on the temperature on day $k$, where $k$ ranges from 0 to $K-1$ ( $K$ being the length of the simulation). 
We assume that $\mathbf{A}$ is time independent and block diagonal:

$$
\mathbf{A}=\left[\begin{array}{cccc}
\mathbf{A}_{1} & \mathbf{0} & \ldots & \mathbf{0} \\
\mathbf{0} & \mathbf{A}_{2} & \ddots & \vdots \\
\vdots & \ddots & \ddots & \vdots \\
\mathbf{0} & \ldots & \ldots & \mathbf{A}_{M}
\end{array}\right]
$$

where $\mathbf{0}$ is a $2 \times 2$ zero matrix and the elements of

$$
\mathbf{A}_{m}=\left[\begin{array}{ll}
a_{\text {max,max }} & a_{\text {max,min }} \\
a_{\text {min,max }} & a_{\text {min,min }}
\end{array}\right]
$$

capture the dependence of maximum and minimum temperature at site $m$ on the prior day's maximum and minimum temperature at site $m$, as indicated by the subscripts (e.g., $a_{\text {min,max }}$ is the dependence of minimum temperature on the preceding day's maximum temperature). With this structure for $\mathbf{A}$, between-site covariance is provided by $\mathbf{C}_{k}$.

We model the time dependence of each component of $\mathbf{B}_{k}$ using harmonics generally written as

$$
\begin{aligned}
b_{k}= & \gamma_{\chi_{k+1}}+\alpha k+\beta_{\chi_{k+1}} \cos (2 \pi k / \tau)+\beta_{\chi_{k+1}}^{\prime} \sin (2 \pi k / \tau) \\
& +\delta_{\chi_{k+1}} \cos (4 \pi k / \tau)+\delta_{\chi_{k+1}}^{\prime} \sin (4 \pi k / \tau)
\end{aligned}
$$

where $\tau$ is the period, assumed to be 365 days. Here, $b_{k}$ is one of the $2^{M}$ entries of $\mathbf{B}_{k}$. Coefficients $\gamma, \alpha, \beta, \beta^{\prime}, \delta$, and $\delta^{\prime}$ are also entries of $2 M \times 1$ vectors. The subscript $\chi_{k+1}$ indicates that $b_{k}$ depends on whether day $k+1$ was wet $(\chi=1)$ or dry $(\chi=0)$.

As in the single-site SHArP, we apply a least squares estimation to determine the parameters in $\mathbf{A}$ and $\mathbf{B}_{k}$. Because there are two temperatures (maximum and minimum) and $\mathbf{A}$ is now a matrix of four elements per site instead of a single coefficient, we minimize the residuals by differentiating with respect to 26 variables per site instead of the 12 in the single-temperature, singlesite case. The $22 M$ resulting equations related to the (POP) parameters of $\mathbf{B}_{k}$ from Eq. (4) are analogous to those presented in Smith et al. (2017) and are omitted for brevity. The remaining $4 M$ equations related to the four elements of $\mathbf{A}$ at each site follow the form

$$
\begin{aligned}
& \sum_{k=0}^{K-1} T_{\max , k}\left(a_{\max , \max } T_{\max , k}+a_{\max , \min } T_{\min , k}\right. \\
& \left.\quad+b_{k, \max }-T_{\max , k+1}\right)=0, \\
& \sum_{k=0}^{K-1} T_{\min , k}\left(a_{\max , \max } T_{\max , k}+a_{\max , \min } T_{\min , k}\right. \\
& \left.\quad+b_{k, \max }-T_{\max , k+1}\right)=0,
\end{aligned}
$$

$$
\begin{aligned}
& \sum_{k=0}^{K-1} T_{\max , k}\left(a_{\min , \max } T_{\max , k}+a_{\min , \min } T_{\min , k}\right. \\
& \left.\quad+b_{k, \min }-T_{\min , k+1}\right)=0, \quad \text { and } \\
& \sum_{k=0}^{K-1} T_{\min , k}\left(a_{\min , \max } T_{\max , k}+a_{\min , \min } T_{\min , k}\right. \\
& \left.\quad+b_{k, \text { min }}-T_{\min , k+1}\right)=0,
\end{aligned}
$$

where $b_{k, \text { max }}$ and $b_{k, \text { min }}$ refer to the elements of $\mathbf{B}_{k}$ that correspond to maximum temperature $T_{\max }$ and minimum temperature $T_{\min }$, respectively. In the case of missing values, summations are taken over available data.

\section{b. Specification of parameters}

In the single-site, single-temperature case, the noise coefficient $\mathbf{c}_{k}$ was a time-dependent vector that depended on whether the day was wet or dry. For multisite SHArP, $\mathbf{C}_{k}$ is a time-dependent, $2 M \times 2 M$ matrix that depends on the spatial POP. In the multisite case, the number of possible POPs is $2^{M}$, which would yield an unmanageably large set of $\mathbf{C}_{k}$ matrices. We reduce this dramatically by performing empirical orthogonal function (EOF) analysis (e.g., Hannachi et al. 2007). Specifically, we calculate the eigenvectors of the $2 M \times 2 M$ spatial covariance matrix of daily occurrence. While we process all days of year together here, it is possible to develop and use seasonal or monthly EOFs. Area weighting was used for EOF analysis, determined via Delaunay triangulation for the station data. For the example application here, we used the positive and negative polarities of the first two EOFs of occurrence to define four POPs (positive polarities are shown in Figs. $2 \mathrm{a}$ and $2 \mathrm{~b}$ ), and then assigned each day to the pattern it most closely resembled as detailed below. The first two EOFs were chosen because they captured the majority of the variance in the domain $(53 \%$ and $8 \%$, respectively). To determine the closest match between a given day's simulated occurrence pattern and the four POPs, the eigenvector of each POP was quantized so that nonnegative components were assigned a value 1 and negative components were assigned a value 0 , and the Euclidian distance was calculated between the quantized eigenvector and the simulated occurrence pattern on that day.

For the study area here, the first quantized eigenvector captured all sites being wet in its positive polarity (Fig. 2a) and all sites being dry in its negative polarity. The second quantized eigenvector captured the mountain sites being wet and the valley sites being dry in its positive polarity (Fig. 2b) and the reverse in its negative polarity. Similar EOFs were found analyzing precipitation occurrence along the statistical downscaling 


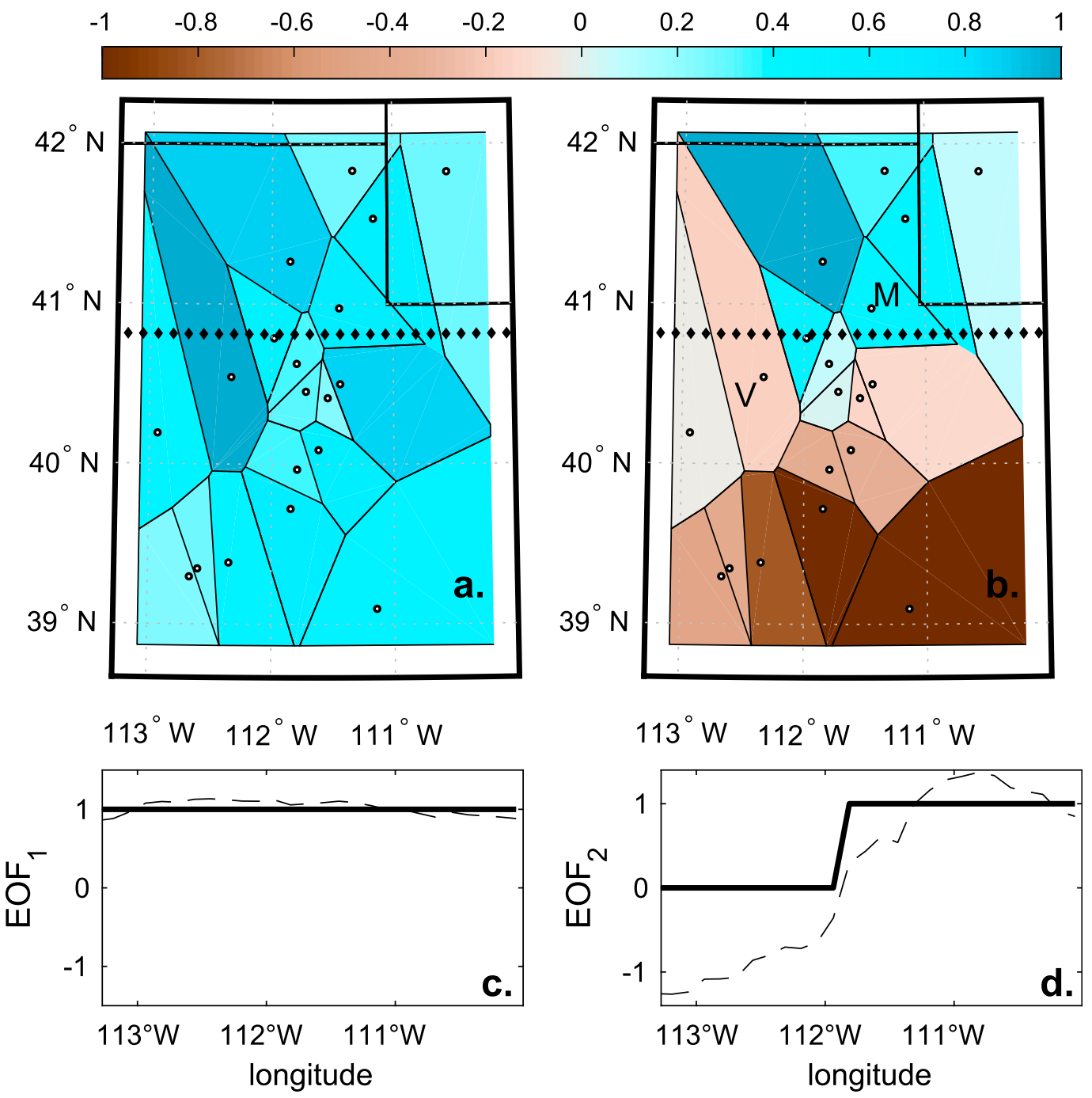

FIG. 2. EOFs of precipitation occurrence: (a) The leading EOF in its positive polarity corresponding to all stations being wet (negative polarity is all stations being dry and is not shown). (b) The second EOF in its positive polarity with mountain stations in the northern part of the domain being wet and lower-elevation stations to the west and south being dry (mountain-wet/valley-dry pattern; negative polarity is the opposite and is not shown). (c),(d) Corresponding first two EOFs diagnosed from statistically downscaled climate model output along the transect of sites indicated by the black diamonds in (a) and (b), respectively; dashed curves are the EOF loading vectors, and solid lines are the quantized versions of the EOFs.

transect (Figs. 2c,d), and these first two EOFs accounted for $62 \%$ and $11 \%$ of the variance for the transect.

We populate each of the four $\mathbf{C}_{k}^{2}$ matrices with the square of residuals $\left(\mathbf{T}_{k+1}-\mathbf{A} \mathbf{T}_{k}-\mathbf{B}_{k}\right)$ specific to the given day of year and one of the four POPs determined via the EOF analysis (there are as many $\mathbf{C}_{k}$ matrices as there are POP EOFs). To ensure sufficient degrees of freedom in the observed record (1950-2005), we sampled the residuals monthly and placed the results on the center day of the month in a matrix $\mathbf{C}_{m}^{2}, m=1, \ldots, 12$. The length and smoothness of the statistically downscaled climate model output (1950-2100) allowed us to populate the matrices daily. We then fit the annual cycles of daily values as in Smith et al. (2017) using Fourier analysis with the general equation

$$
\begin{aligned}
c_{k}^{2}= & \rho+\epsilon \cos (2 \pi k / \tau)+\epsilon^{\prime} \sin (2 \pi k / \tau) \\
& +\kappa \cos (4 \pi k / \tau)+\kappa^{\prime} \sin (4 \pi k / \tau),
\end{aligned}
$$

where $c_{k}^{2}$ is a time-dependent element of one of the four $\mathbf{C}_{k}^{2}$ matrices. On average, the harmonic fits account for $85 \%$ of the variance in the monthly values. We then take the principal square root to yield the four $\mathbf{C}_{k}$ matrices. During estimation and simulation, 

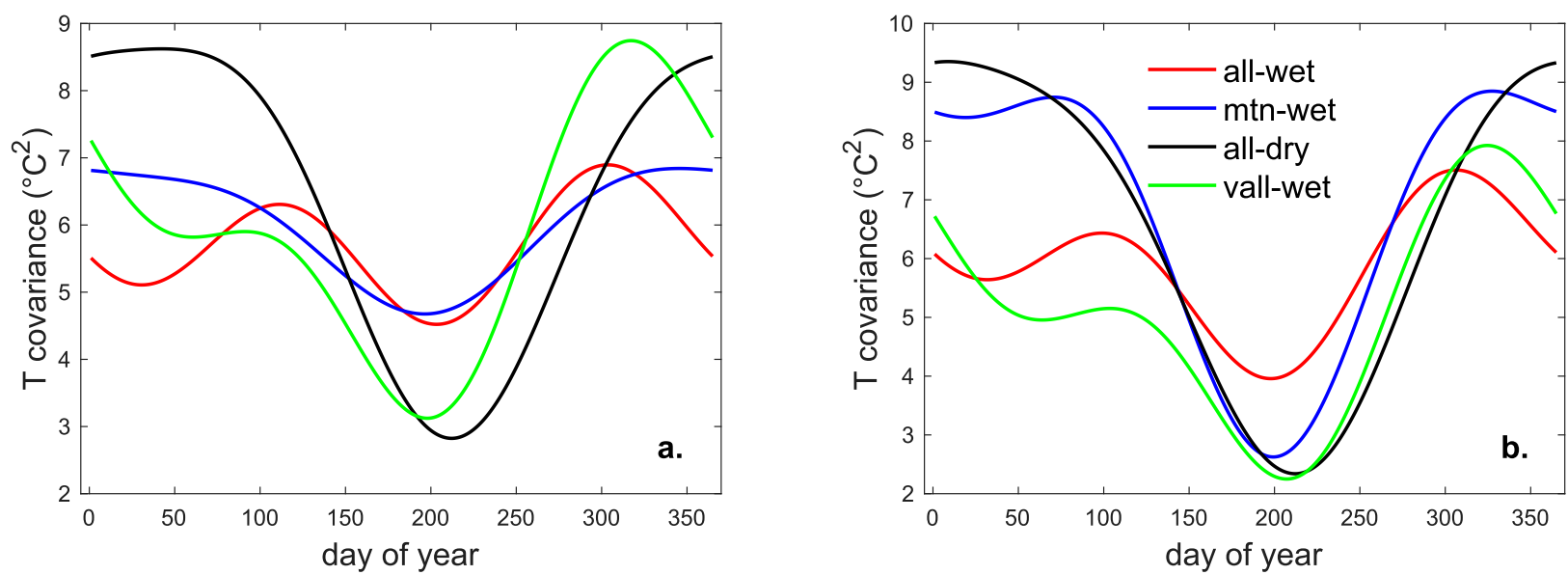

FIG. 3. Average covariance of maximum temperature residuals (a) between the valley site marked V in Fig. $2 \mathrm{~b}$ and the blue-shaded sites in Fig. $2 b$ and (b) between the mountain site marked $M$ in Fig. $2 b$ and the brown-shaded sites in Fig. 2b. Abbreviations in the legend indicate, in order, all-wet, mountain-wet/valley-dry, all-dry, and valley-wet/mountain-dry precipitation-occurrence patterns.

each of the $\mathbf{C}_{k}$ matrices contains maximum and minimum temperature for each site, so they are of size $2 M \times 2 M$ with row 1 corresponding to maximum temperature at site 1 , row 2 corresponding to minimum temperature at site 1 , row 3 corresponding to maximum temperature at site 2 , and so on. Once we have determined the parameters in $\mathbf{A}, \mathbf{B}_{k}$, and $\mathbf{C}_{k}$, we simulate maximum and minimum temperature simultaneously at all sites.

\section{Model significance, validation, and illustration}

\section{a. Model significance}

The POP-specific covariance patterns are one of the principal innovations in the multisite generalization of SHArP, so we provide some statistical analysis to emphasize their importance. As noted at the end of section $3 b$, for each month we have four $\mathbf{C}_{m}^{2}$ matrices-one for each POP. We tested the null hypothesis that these four covariance matrices are equal using Box's $M$ test, which is a $\chi^{2}$ approximation of the modified likelihood-ratio statistic (e.g., Rencher and Christensen 2012). For all 12 months, we can reject at the $95 \%$ confidence level the null hypothesis of covariance matrix equality, meaning the POP-based decomposition captures statistically significant differences in observed temperature covariance. We can also reject at the $95 \%$ confidence level the null hypotheses of covariance matrix equality for subsets such as all-wet and mountainwet/valley-dry regimes.

To depict some of the patterns underlying these hypothesis testing results, we average over elements of the $\mathbf{C}_{k}^{2}$ matrices and plot their annual cycles, recalling that the harmonic fits in these $\mathbf{C}_{k}^{2}$ matrices account on average for
$85 \%$ of observed covariance. We begin with the covariance of maximum temperature residuals between the valley site marked $\mathrm{V}$ in Fig. $2 \mathrm{~b}$ and blue-shaded sites in Fig. 2b, and Fig. 3a shows the average of these covariances for each day of the year. Covariation is stronger in the cold season, and all-dry values are approximately $50 \%$ larger than all-wet values in January, with mountainwet and valley-wet values being intermediate. As precipitation dynamics make the transition to monsoonal convective regimes during the warm season, the all-wet and mountain-wet regimes have larger covariances than do the all-dry and valley-wet regimes. Examining covariance of maximum temperature residuals between the mountain site marked $M$ in Fig. $2 b$ and brown-shaded sites in Fig. 2b, we find similar strong and seasonally evolving separation between the all-wet and all-dry regimes, with the mountain-wet regimes tracking more closely with all-dry regimes in this case (Fig. 3b).

\section{b. Model validation and illustrative patterns}

The model skill and validation at individual sites was presented in Smith et al. (2017), so we focus on the multisite aspects of the performance here based on results from the historical station observations. Performance for the historical and future statistically downscaled data was also assessed and found to be comparable or better (not shown), consistent with the downscaling producing smoother variations in space and time compared to station observations.

We begin with Fig. 4 to illustrate the model's fidelity in capturing spatial extrema of daily minimum and maximum temperature. Shown are quantile-quantile plots of the spatial maximum of $T_{\max }$, minimum of $T_{\min }$, maximum of $T_{\min }$, and minimum of $T_{\max }$. 

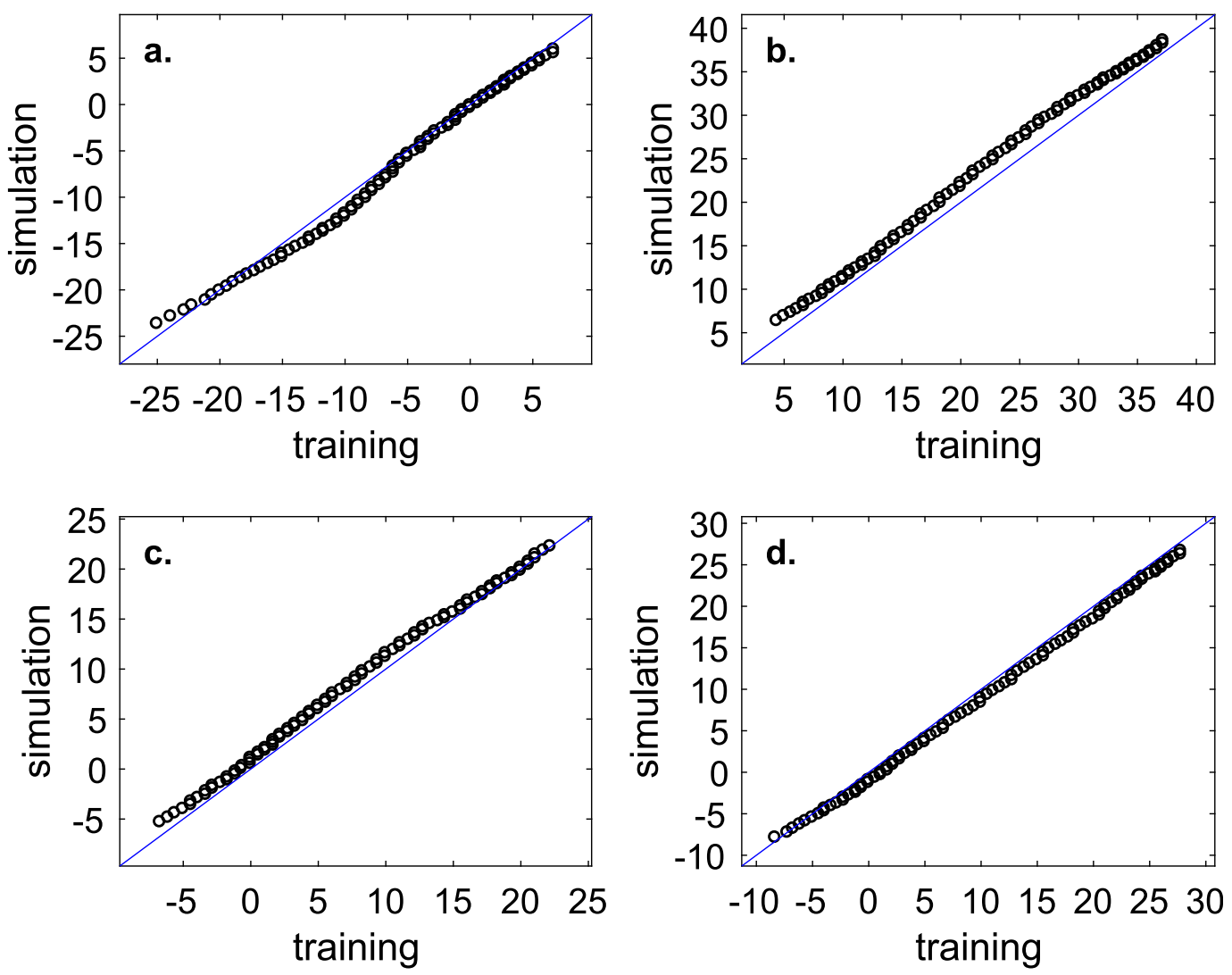

FIG. 4. Quantile-quantile plots for temperature spatial extrema using daily data from all months: (a) minimum of $T_{\min }$, (b) maximum of $T_{\max }$, (c) maximum of $T_{\min }$, and (d) minimum of $T_{\max }$. Blue lines are 1:1 lines.

SHArP tends to slightly overpredict the maximum extrema (Figs. 4b,c) and slightly underpredict the minimum extrema (Figs. 4a,d), but the agreement is very good overall.

Segregating by POP, SHArP captures seasonal contrasts in mean $T_{\max }$ and $T_{\min }$ well (Figs. 5a-d). For pairwise, between-station covariation, SHArP captures aspects of the seasonal changes such as the smaller covariances in summer and larger covariances in the transition seasons (Figs. 5e-h). Large changes in the position of a given seasonal cluster between panels in Fig. 5 illustrate the importance of the POPs in modulating intersite covariation. A portion of the covariance in Figs. $5 \mathrm{e}-\mathrm{h}$ is associated with changes in mean temperature, so we also show the same analysis calculated for the stochastic residuals $\left(\mathbf{T}_{k+1}-\mathbf{A} \mathbf{T}_{k}-\mathbf{B}_{k}\right)$ in Figs. 5i-1. Deviations tend toward the right of the 1:1 line, indicating conditions under which SHArP tends to underpredict covariation. One example is winter temperatures during the all-dry POP (Fig. 5j), and some of the strong observed covariation may stem from persistent cold-air-pool events (Lareau et al. 2013).
To further illustrate the utility of the POP-based matrices $\mathbf{C}_{k}$, Fig. 6 contrasts spatial patterns of temperature covariance between different POPs for selected seasons. Strong variations in longitude are in part due to collapsing the latitude dimension of the data for display, and SHArP captures these spatial patterns well. During autumn, maximum temperatures near the peak of the Wasatch Range covary strongly with values along the western face of the range, and these covariances are almost uniformly increased in the mountain-wet results relative to all-wet results (Fig. 6a). During spring, minimum temperatures over the high elevations in the eastern portion of the domain covary more strongly with values to the east, and SHArP captures the westward decrease in covariance moving down in elevation (Fig. 6b). Also, for spring minimum temperatures, SHArP captures the tendency for all-dry days to have larger covariance than all-wet days (Fig. 6b).

We close this section with some remarks and results for simulation of future temperature variations, including trends. As noted above, SHArP trained on statistically downscaled climate model output along the transect in Fig. 1 produces similar POP EOFs to those 

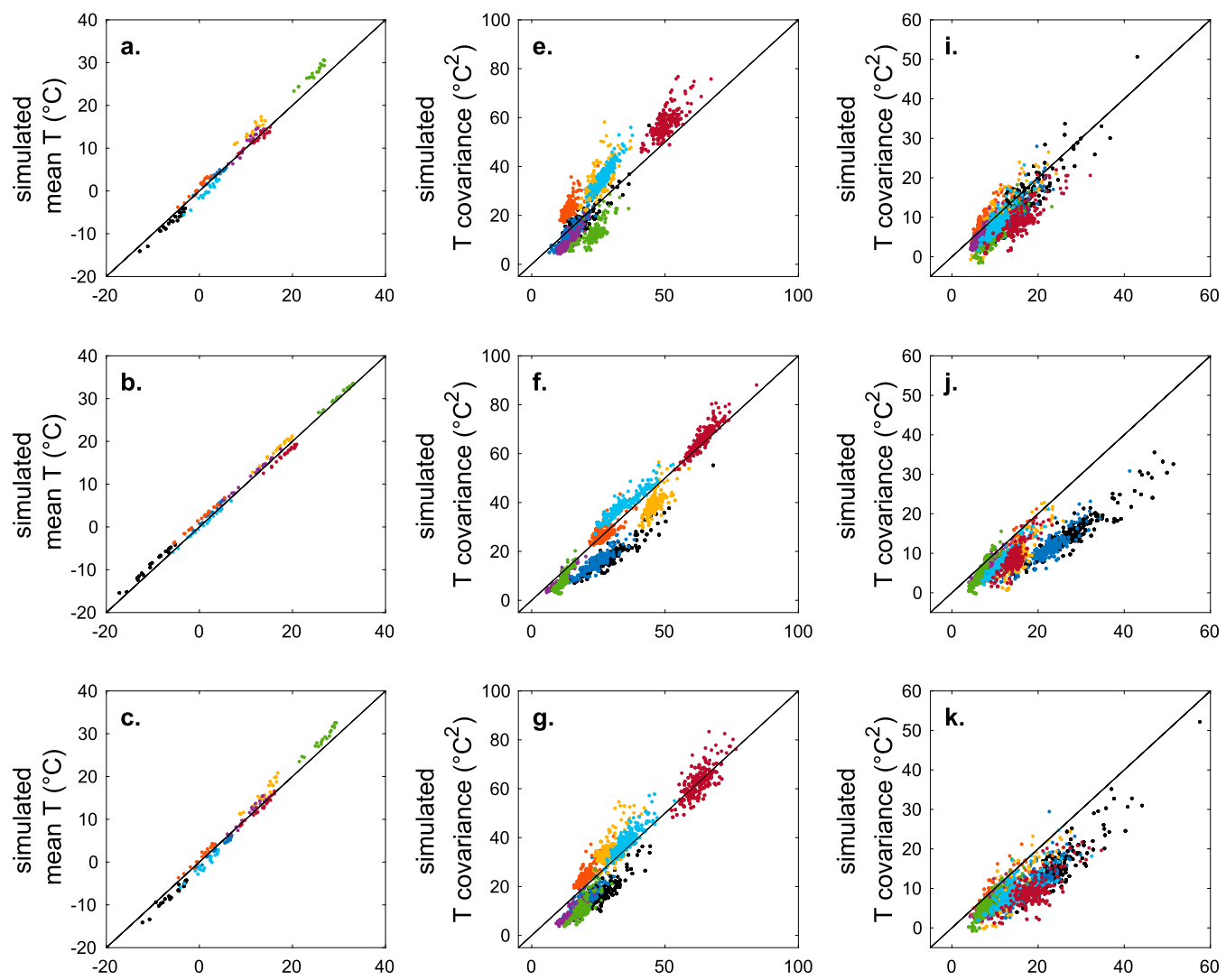

- winter $T_{\text {min }}$

- spring $T_{\min }$

spring $T_{\max }$

- summer $T_{\min }$

- summer $T_{\max }$

fall $T_{\min }$

- fall $T_{\max }$
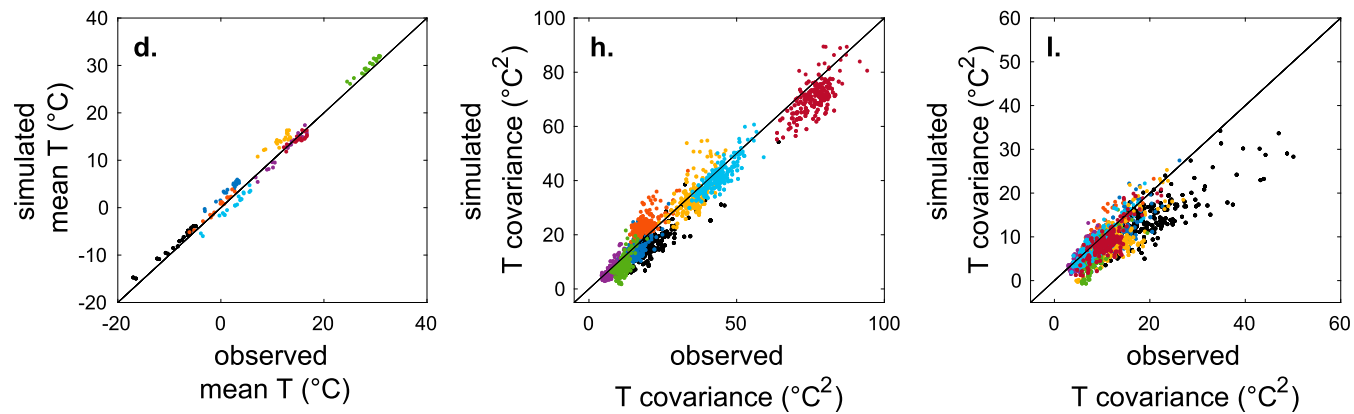

FIG. 5. Model assessment on the basis of station observations with results segregated by four precipitation-occurrence patterns: (top) allwet, (top middle) all-dry, (bottom middle) mountain-wet/valley-dry, and (bottom) valley-wet/mountain-dry regimes for the statistics of (a)-(d) mean temperature, (e)-(h) pairwise between-station covariance of temperature, and (i)-(l) pairwise between-station covariance of temperature residuals. Black lines are 1:1 lines.

found for station data (Fig. 2) and comparable or better validation due to data smoothness. Training SHArP on the statistically downscaled data for 1950-2100 enables us to simulate future variations. As an example, annual cycles of maximum temperature are shown for four sites corresponding to a late-century year in Figs. 7a and 7b. SHArP, in addition to simulating realistic annual cycles and variance at each site, provides realistic intersite covariation that is temporally synchronized by POPs. SHArP is also able to capture long-term trends with realistic intersite covariation as illustrated by annual mean minimum temperatures at the four sites (cf. Figs. 7c,d).

\section{Multisite simulation of daily precipitation}

\section{a. Formulation and parameter estimation for precipitation occurrence}

The precipitation model we use with SHArP largely follows formulations presented in Woolhiser (2008) and Wilks (2009), except we introduce a trend term in the perturbation of the Markov chain precipitation-occurrence 


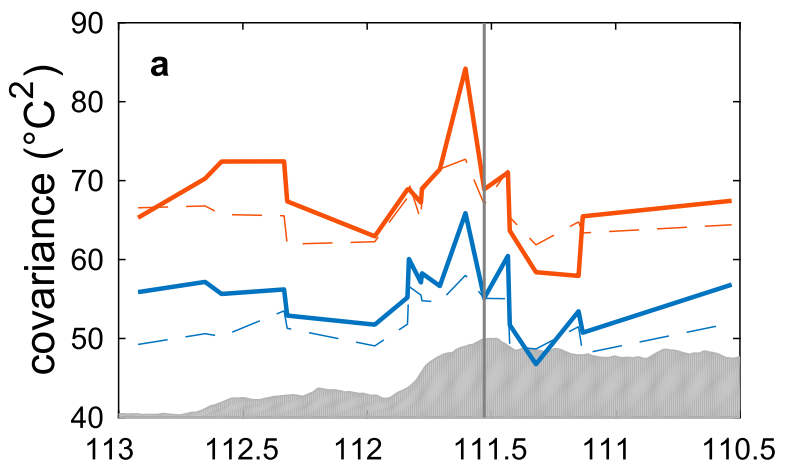

\section{- - all-wet training \\ - - mountain wet training all-wet simulation mountain-wet simulation terrain}

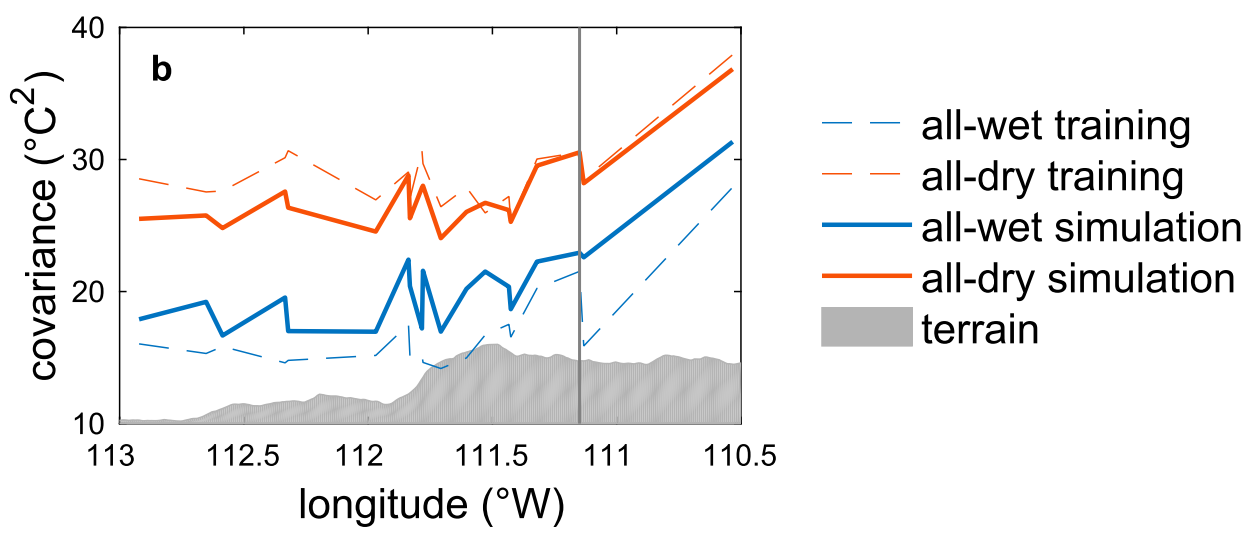

FIG. 6. (a) Covariance of maximum temperature between station $13\left(40.08^{\circ} \mathrm{N}, 111.60^{\circ} \mathrm{W}\right)$ and all other stations for days in autumn that had all-wet (blue) or mountain-wet/valley-dry (red) values. The longitude of station 13 is indicated by the vertical gray line. (b) Covariance of minimum temperature between station $17\left(41.82^{\circ} \mathrm{N}, 110.53^{\circ} \mathrm{W}\right)$ and all other stations for days in spring that had all-wet (blue) or all-dry (red) values. The longitude of station 17 is indicated by the vertical gray line. Gray shading indicates relative terrain height averaged over the latitude range in Fig. 1.

probabilities so that the framework can simulate climate change. We provide details leading up to the introduction of the trend here for completeness.

We model precipitation occurrence with a two-state (wet or dry), second-order Markov chain such that the probability of precipitation on any given day depends on the precipitation state on the previous two days:

$p_{i j 1}(t)=P\left\{\chi_{t}=1 \mid \chi_{t-1}=j, \chi_{t-2}=i\right\} ; \quad t=1,2, \ldots, 365 Y$,

where $Y$ indicates the number of years. We use a secondorder Markov chain as opposed to a first-order Markov chain because the former has been shown to better capture the occurrence of dry spells (e.g., Stern and Coe 1984; Wilks 1999a), which are common in the semiarid region in this study. The unperturbed probability time series in Eq. (6) are cyclostationary, written as inverse logits, and found via maximum likelihood using a Newton-Raphson iterative procedure (Woolhiser 2008). To illustrate the spatiotemporal patterns of these probability functions in the statistically downscaled climate model output, the $p_{011}$ values for each site over any given year are shown in
Fig. 8a. The probability of precipitation is overall higher in the mountains than in the valleys, and the maximum in $p_{011}$ for most sites occurs near day of year 100 . The marked increase in $p_{011}$ at the valley-to-mountain transition near site 15 motivates the use of EOF analysis to categorize the POPs and contributes to mountain versus valley contrast captured by EOF 2 (recall Figs. 2b,d).

We generalize the precipitation-occurrence process to $m=1, \ldots, M$ sites by defining the multisite occurrence (Wilks 2009):

$$
\chi_{t}(m)= \begin{cases}1 & \text { if } w_{t}(m) \leq \Phi^{-1}\left[p_{i j 0}(t)\right] \\ 0 & \text { otherwise }\end{cases}
$$

where $\Phi^{-1}[]$ is the probit function and $w_{t}(n) \sim \mathrm{N}[0,1]$ is Gaussian white noise. To achieve spatially coherent precipitation occurrence, the Markov chain model in Eq. (7) is forced by a vector of mutually correlated standard Gaussian variates $\mathbf{w}_{t}$ that is characterized by correlation matrix $\mathbf{C}_{R}$. We populate $\mathbf{C}_{R}$ so that the synthetic correlation matrix $\mathbf{C}_{\chi}$ matches its observed counterpart $\mathbf{C}_{x}$. We achieve this via brute-force iteration (Brissette et al. 2007): 

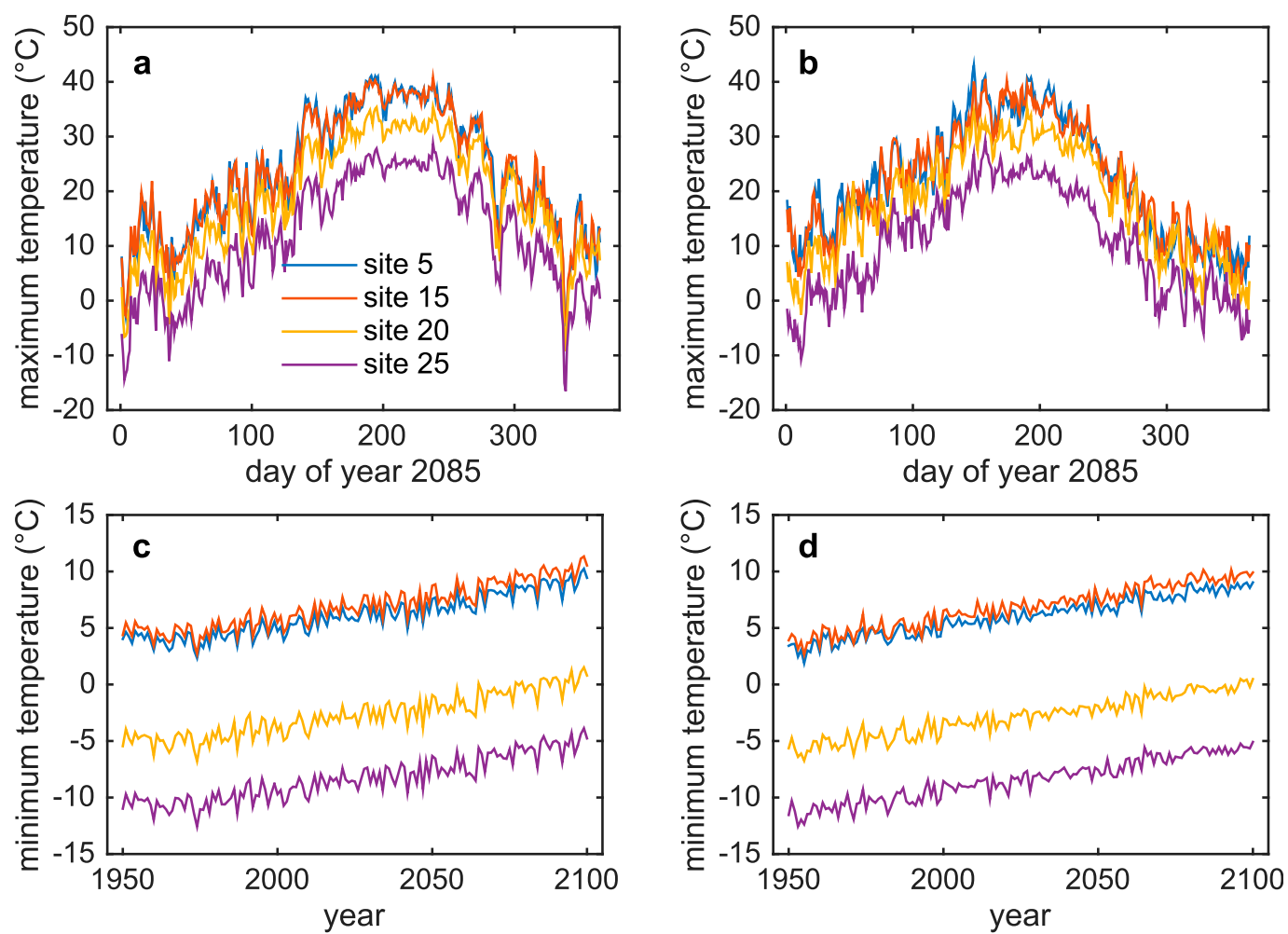

FIG. 7. Daily maximum temperature in 2085 at four sites for (a) statistically downscaled climate model output and (b) a sample realization from SHArP. Also shown are annual mean minimum temperature from (c) statistically downscaled climate model output and (d) SHArP at the same four sites. Sites are numbered 1-30 from west to east in Fig. 1.

$$
\mathbf{C}_{R}(i+1)=\mathbf{C}_{R}(i)+\eta\left(\mathbf{C}_{x}-\mathbf{C}_{\chi}\right)
$$

with initial guess $\mathbf{C}_{R}(1)=\mathbf{C}_{x}, \eta=0.1$, and $\sim 30$ iterations to achieve $10^{-3}$ precision.

\section{b. Formulation and parameter estimation for precipitation amount}

In addition, we model precipitation amount using a mixed exponential distribution following Wilks (1999b). The associated probability density function

$f[r(m)]=\frac{\alpha}{\beta_{2}(m)} \exp \left[\frac{-r(m)}{\beta_{2}(m)}\right]+\frac{1-\alpha}{\beta_{1}(m)} \exp \left[\frac{-r(m)}{\beta_{1}(m)}\right]$

is the sum of two exponential distributions; the first with larger mean $\beta_{2}$ occurs with probability $\alpha$, and the second with smaller mean $\beta_{1}$ occurs with probability $1-\alpha$. When precipitation occurs at site $m$, we choose between $\beta_{1}$ and $\beta_{2}$ according to

$$
\beta_{t}(m)=\left\{\begin{array}{ll}
\beta_{2}(m) & \text { if } \frac{\Phi\left[w_{t}(m)\right]}{p_{i j 1}(m)} \leq \alpha \\
\beta_{1}(m) & \text { otherwise }
\end{array},\right.
$$

where $p_{i j 1}(m)$ is the appropriate transition probability from Eq. (6). The formulation in Eq. (10) captures the tendency for larger precipitation amounts to occur near the interior of wet areas because, for stations and days with small $w_{t}(m)$ [i.e., first line of Eq. (10)], other stations around the site are likely to be wet because of the spatial autocorrelation in $\mathbf{C}_{R}$, and the larger precipitation mean $\left(\beta_{2}\right)$ is selected (Wilks 1999a,b). Spatiotemporal variations in these amount parameters for the study region are shown in Fig. 8, illustrating that the mixed exponential means $\left(\beta_{1}, \beta_{2}\right)$ tend to be larger in the mountains and outside of summer and that the probability of selecting the larger mean $(\alpha)$ tends to maximize in spring and minimize in summer.

The amount is then recovered from the probability density function via

$$
r_{t}(m)=h-\beta_{t}(m) \ln [\nu(m)],
$$

where $h$ is the precipitation-occurrence threshold (defined here as $0.254 \mathrm{~mm}$ ) and $\nu(m) \sim U[0,1]$ is uniformly distributed. The required spatial correlation for $\nu(m)$ is achieved via brute-force iteration (Brissette et al. 2007) analogous to the determination of $\mathbf{C}_{R}$ as described 

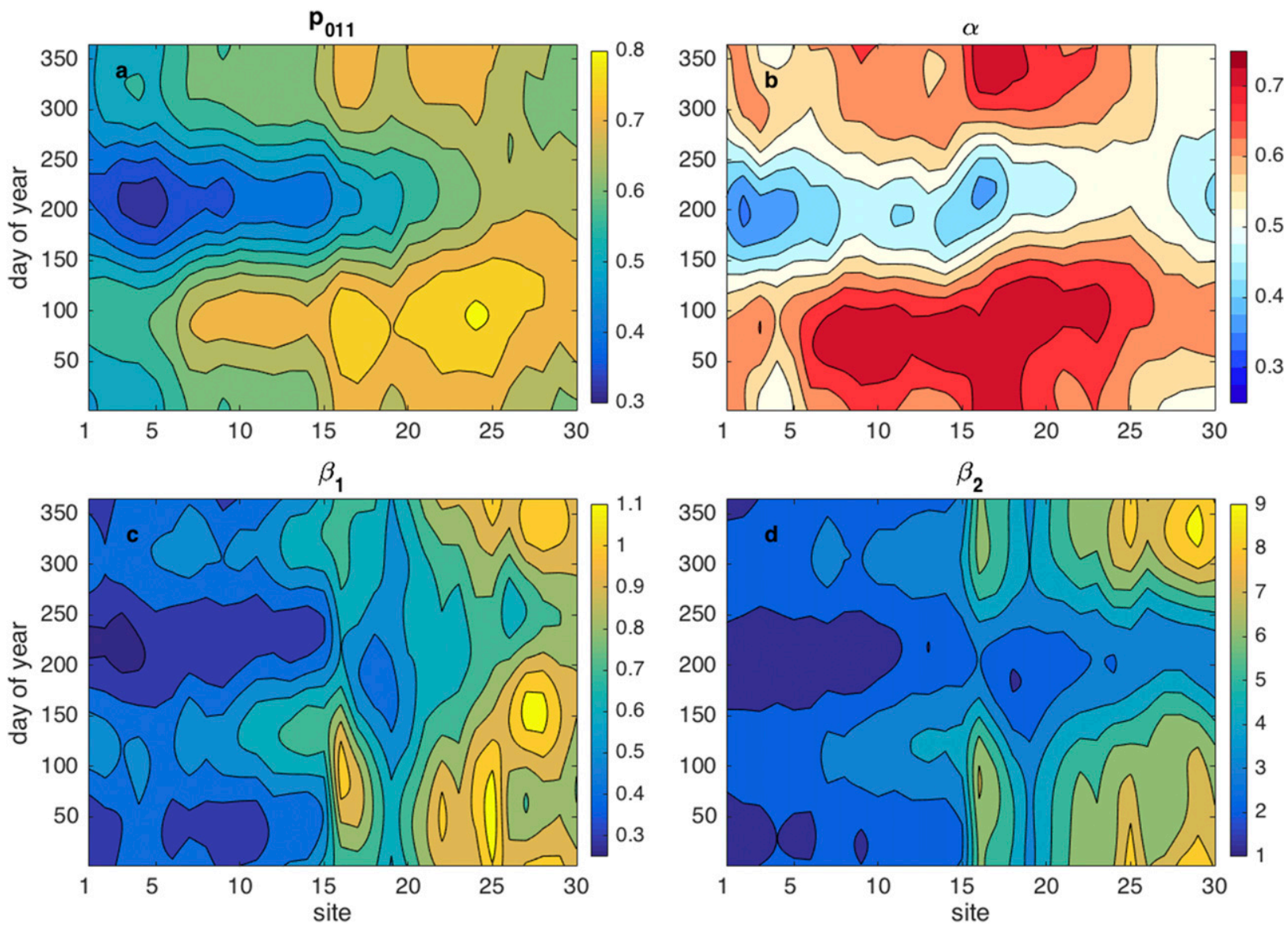

FIG. 8. For sites 1-30 along the statistical downscaling transect: (a) raw (nonperturbed) probability of precipitation given that the preceding two days were dry and wet, respectively, (b) the probability $\alpha$ of selecting the higher precipitation mean from the mixed exponential precipitation distribution, (c) the lower mean from the mixed exponential precipitation distribution $\left(\beta_{1}\right.$; mm), and (d) the raw (nonperturbed) higher mean from the mixed exponential precipitation distribution $\left(\beta_{2} ; \mathrm{mm}\right)$. Note the different scales for $\beta_{1}$ and $\beta_{2}$.

before with regard to multisite occurrence. Similar to the probability of precipitation, the means $\beta_{1}$ and $\beta_{2}$ increase markedly at the valley-to-mountain transition near site 15 (Figs. 8c,d), and they tend to be smaller in the summer months. The probability of choosing the larger mean $(\alpha)$ is smallest in the summer months for all sites along the transect (Fig. 8b).

\section{c. Formulation and parameter estimation for climate perturbation}

Because of the likely effects of climate change in the future, especially relating to the snowpack in the western United States (e.g., Mote 2006), we modified the precipitation components of SHArP to include these effects. We use the formulation for simulating precipitation occurrence introduced in Smith et al. (2017), where we define perturbed versions of the $p_{i j k}$ values that can incorporate trends and sensitivity to teleconnection indices, extending ideas presented in Woolhiser (2008). In addition, the larger mean in the mixed exponential precipitation amount distribution $\left(\beta_{2}\right)$ is here allowed to have a trend and dependence on teleconnection indices, analogous to the perturbed formulation of $p_{i j 1}$.

The perturbed $p_{i j 1}$ values are given by

$p_{i j 1}^{\prime}(t)=p_{i j 1}(t)+\gamma_{0}^{i j 1}+\gamma_{1}^{i j 1} t+\gamma_{2}^{i j 1} E\left(t-\tau_{E}\right)+\gamma_{3}^{i j 1} P\left(t-\tau_{P}\right)$,

where the $\gamma_{0,1}$ coefficients enable a trend and the $\gamma_{2,3}$ coefficients provide potentially time-lagged sensitivity to climate variability modes, here chosen to be ENSO $(E)$ and PDO $(P)$. The perturbation of $\beta_{2}$ is formulated analogously. The $\gamma$ parameters in Eq. (12) are determined via maximum likelihood in a stepwise fashion, first bringing in the trend, then adding the first oceanic mode, and finally adding the second oceanic mode. At each step, we use the Akaike information criterion to include only parameters that significantly improve the logarithmic likelihood. We use ENSO and PDO indices here because these modes are important in influencing 

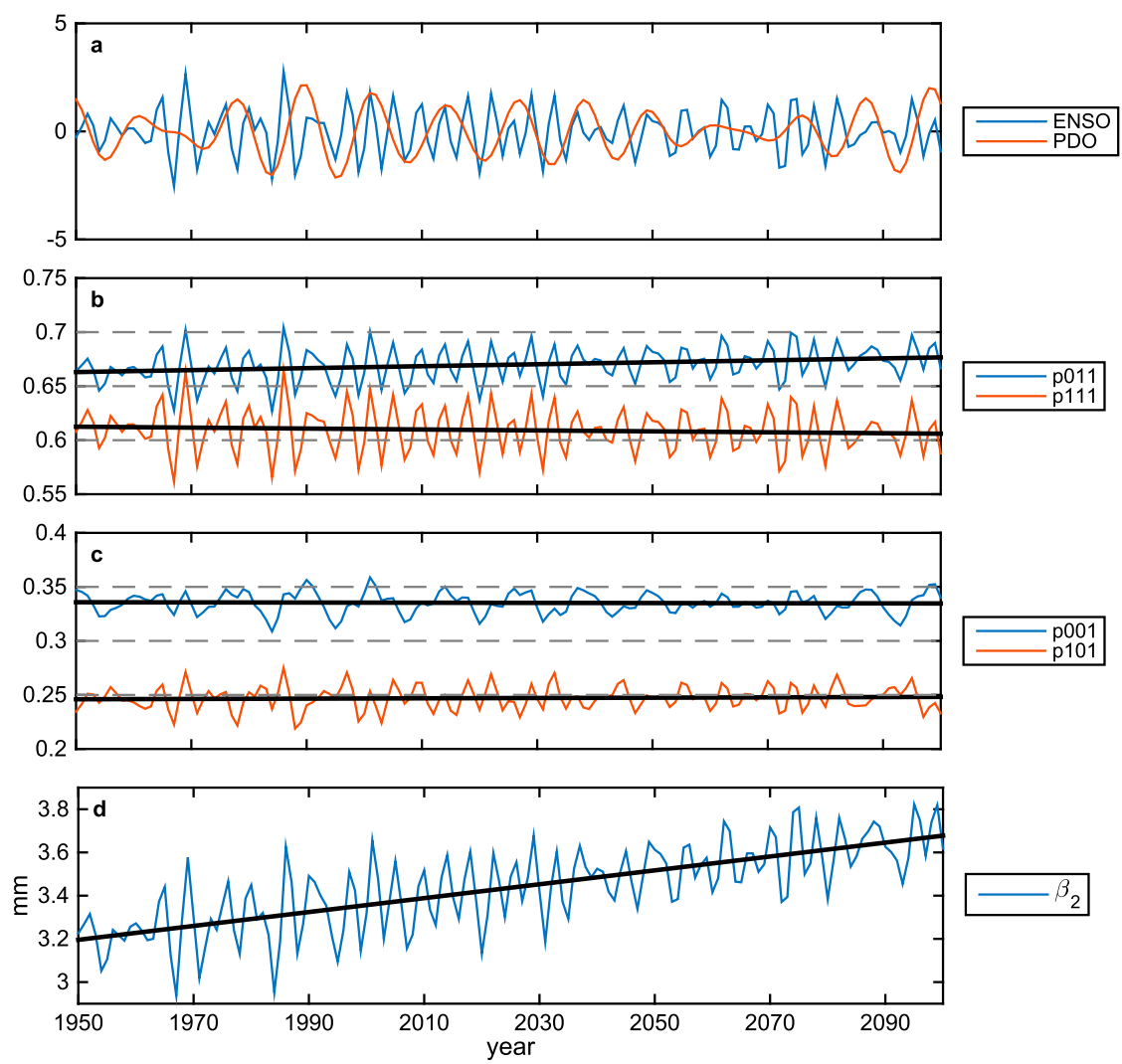

FIG. 9. (a) Standardized indices of the oceanic modes of variability (ENSO and PDO) diagnosed from CCSM4. For the site nearest KSLC, (b), (c) annual mean perturbed $p_{i j 1}$ values with trend lines indicated in black, and (d) annual mean perturbed $\beta_{2}$ values.

precipitation variability in the study region (e.g., Wise 2010), CCSM4 captures these effects well (Smith et al. 2015), and their inclusion significantly increases the log likelihood. Nonetheless, this is just an illustration of capability and not a claim that complicated ocean-toGreat Basin teleconnection dynamics can be fully captured by index-based perturbation of parameters.

As an illustrative example, the perturbed $p_{i j 1}^{\prime}$ values for the statistical downscaling grid point nearest KSLC over the analysis period (1950-2100) are shown in Figs. $9 \mathrm{~b}$ and $9 \mathrm{c}$, and an increase in $p_{111}$ is visible over the record. In addition, there is salient periodic variability in all of the $p_{i j 1}$ time series following the oceanic forcing terms (cf. Figs. 9b,c with Fig. 9a). Determining the perturbation of $\beta_{2}$ via maximum likelihood formulated analogous to Eq. (12) yielded sensitivity to the oceanic modes with a clear increasing trend over the record (Fig. 9d).

We simulated multisite daily precipitation 500 times from 1950 to 2100 to illustrate variability in total precipitation from year to year. Figure 10 shows this variability at KSLC in comparison with the training data. Note the overall increasing trend and low-frequency variability due to ENSO and the PDO. The tendency for correlation between the training data and the ensemble mean arises because the oceanic modes $(E$ and $P$ ) driving the precipitation occurrence and amount were diagnosed from the coupled global climate model simulation that produced the training data.

\section{Discussion and conclusions}

We extended the stochastic temperature simulation framework introduced by Smith et al. (2017) by generalizing the single-temperature, single-site formulation to encompass maximum and minimum temperatures correlated between multiple sites. This study focused substantially on the temperature component because temperature has received comparatively less attention in the literature despite its importance to snowpack variability, and we were able to markedly improve its realism by leveraging techniques described in Smith et al. (2017). The formulation of SHArP shares conceptual similarity with some recent GLM frameworks referenced in the introduction, but it is unique in its incorporation of precipitation spatial pattern effects (e.g., mountain-wet/valley-dry patterns) on temperature 


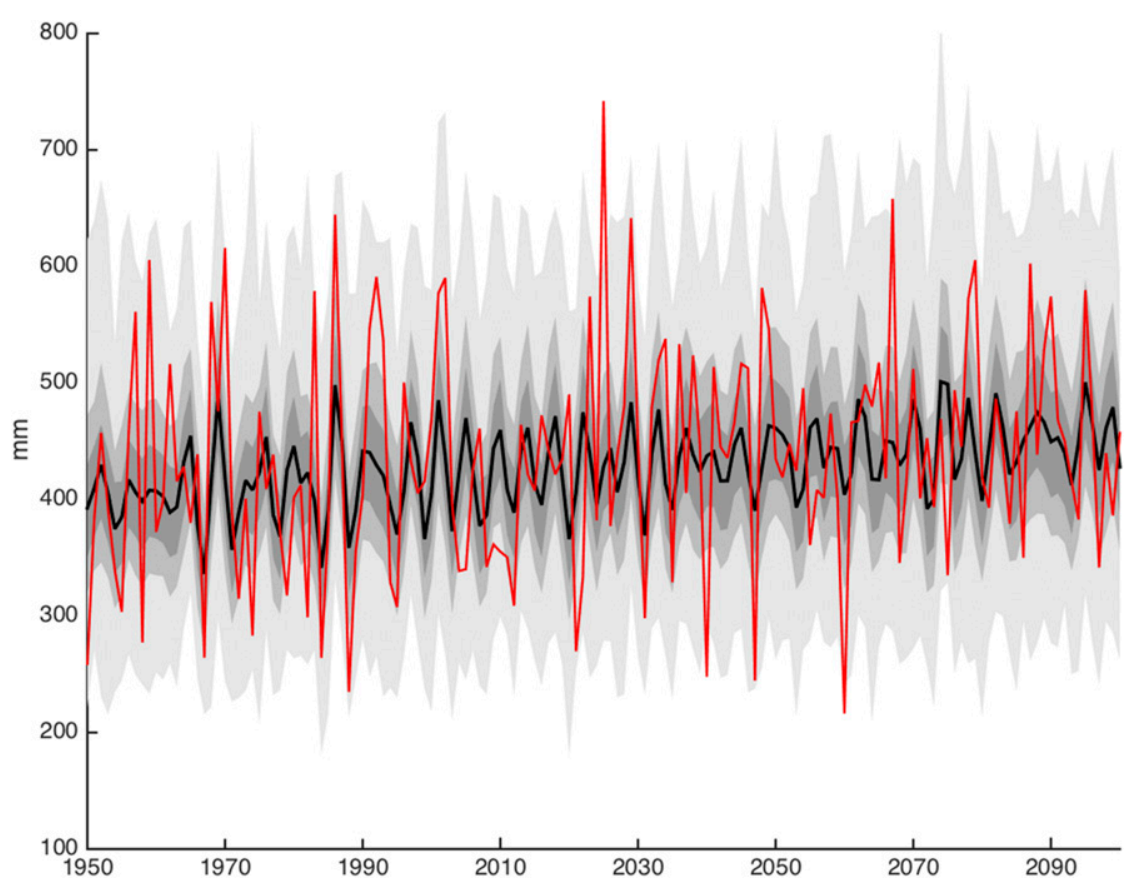

FIG. 10. Annual total precipitation for site nearest KSLC over the period 1950-2100. The mean of the data is shown by the solid black curve, and degrees of shading from darker to lighter indicate the 25th-75th percentiles, the 10th-90th percentiles, and the range. The total number of simulations is 500. The training data from BCCA CCSM4 are shown in red.

temporal evolution and spatial covariance. Hypothesis testing shows that the POP-based decomposition captures statistically significant differences in observed temperature covariance. In addition, we presented a compatible multisite daily precipitation simulation framework based on Markov chain ideas introduced by Woolhiser (2008) and Wilks $(1998,2009)$. The precipitation framework can capture lagged dependence on climate modes such as ENSO and PDO and was generalized here to capture trends associated with climate change. This study used data from weather station locations and grid points on the downscaled model output grid. Data can be generated for "unobserved" or off-grid locations by interpolating the model parameters using elevation and distance relationships via, for example, kriging or use of distance and elevation dependencies following Wilks (1999b).

A key advance from the mathematical formulation of single-site SHArP introduced in Smith et al. (2017) is the change from temporally varying noise coefficient vectors (one for dry days and one for wet days) to noise coefficient matrices that depend on the multisite spatial POP and simulate observed intersite correlations in temperature. A total of $M$ sites yields an unmanageably large $2^{M}$ possible POPs, so we employ EOF analysis to reduce the number of possible POPs to some number much smaller than $2^{M}$. Here, we used the leading two EOFs for illustration, with the first capturing the contrast between all sites wet and all sites dry, and the second capturing mountain-wet/valley-dry versus mountain-dry/ valley-wet patterns. The number of EOFs used might be increased depending on the patterns of variability in a particular study region, but it needs to be balanced against the accompanying decrease in sample size for estimation of the covariance matrices. After the residual error for each day is assigned to one of four noise coefficient matrices $\mathbf{C}_{k}$, the entries in the matrices are temporally smoothed via Fourier analysis, and those curves are used in the generation process. This method recognizes only a subset of the total variance in spatial patterns of occurrence, but we found that two harmonics were sufficient for capturing the statistical properties of the input data, and use of more harmonics changed the results minimally. By modeling POP-specific residuals, SHArP is able to capture statistically significant contrasts between precipitation regimes associated with large versus small intersite temperature covariance.

Another key change associated with the multisite generalization is related to the $\mathbf{A}$ matrix in Eq. (1), which replaces the scalar $a$ in the single-site, single-temperature case. The matrix $\mathbf{A}$ contributes to the autocorrelation of $T_{\max }$ and minimum temperature $T_{\min }$ at each site, and is block diagonal so it provides no intersite effects. More specifically, A has four entries for each site: the dependence of $T_{\max }$ on the previous day's $T_{\max }$ and $T_{\min }$, 
and the dependence of $T_{\min }$ on the previous day's $T_{\min }$ and $T_{\max }$. The dependence of $T_{\max }$ on the previous day's $T_{\min }$ is arguably the least physical of the four relationships and could be omitted if weak. The block diagonal assumption on $\mathbf{A}$ means that all intersite correlation is handled by the noise $\mathbf{C}_{k}$ matrices described above, and is consistent with block diagonal assumptions made for the matrices that control the autocorrelation of temperature noise in versions of the Richardson model presented in previous studies (e.g., Richardson 1981; Wilks 1999b, 2009).

Testing the encoding of SHArP, we verified that the model accurately estimates the parameters of a broad range of synthetic multisite input data that we generated using Eq. (1) (i.e., the estimation procedures recover $\mathbf{A}_{k}$, $\mathbf{B}_{k}$, and $\mathbf{C}_{k}$ ). Tested examples include the full model in Eq. (1) and also simplifications such a vector autoregressive process $\left(\mathbf{B}_{k}=0\right)$ with temporally invariant $\mathbf{C}_{k}$, or $\mathbf{C}_{k}$ matrices populated with smooth harmonic time series. Diagnostics such as intersite correlation matrices are also skillfully recovered by SHArP when the training data are generated consistent with its model formulation. Similar to other weather generators, though, the performance diagnostics of SHArP can of course degrade for processes with prominent components (e.g., nonlinearities) not captured by its basic formulation. As an example, SHArP currently assumes $\mathbf{A}_{k}$ does not depend on time, so generating synthetic training data using Eq. (1) with marked temporal fluctuations in $\mathbf{A}_{k}$ produces positive or negative discrepancies in intersite correlation at different times during the annual cycle. The actual training data used here certainly contain variability associated with processes not in the SHArP formulations. Nonetheless, fitting SHArP to these data yields intersite squared correlations that match the corresponding training data diagnostics to within 0.05 , suggesting that the formulation in Eq. (1) is sufficiently complex while still being parsimonious.

Acknowledgments. This material is based upon work supported by the National Science Foundation under grants EPS-1135482, EPS-1135483, EPS-1208732, and DMS-1407574. Any opinions, findings, and conclusions or recommendations expressed in this material are those of the authors and do not necessarily reflect the views of the National Science Foundation. Provision of computer infrastructure by the Center for High Performance Computing at the University of Utah is gratefully acknowledged. We acknowledge the World Climate Research Programme's Working Group on Coupled Modelling, which is responsible for CMIP, and we thank the climate modeling groups for producing and making available their model output. For CMIP, the U.S. Department of Energy's Program for Climate Model
Diagnosis and Intercomparison provides coordinating support and led development of software infrastructure in partnership with the Global Organization for Earth System Science Portals. CPC U.S. Unified Precipitation data were provided by the NOAA/OAR/ESRL Physical Sciences Division (https://www.esrl.noaa.gov/psd/).

\section{REFERENCES}

Brissette, F., M. Khalili, and R. Leconte, 2007: Efficient stochastic generation of multi-site synthetic precipitation data. J. Hydrol., 345, 121-133, https://doi.org/10.1016/j.jhydrol.2007.06.035.

Bureau of Reclamation, 2013: Downscaled CMIP3 and CMIP5 climate and hydrology projections: Release of downscaled CMIP5 climate projections, comparison with preceding information, and summary of user needs. Bureau of Reclamation Tech. Rep., 47 pp. + appendixes, https://gdo-dcp.ucllnl. org/downscaled_cmip_projections/techmemo/downscaled_ climate.pdf.

Caraway, N. M., J. L. McCreight, and B. Rajagopalan, 2014: Multisite stochastic weather generation using cluster analysis and k-nearest neighbor time series resampling. J. Hydrol., 508, 197-213, https://doi.org/10.1016/j.jhydrol.2013.10.054.

Chen, J., F. Brissette, and X. Zhang, 2014: A multi-site stochastic weather generator for daily precipitation and temperature. Trans. ASABE, 57, 1375-1391, https://doi.org/ $10.13031 /$ trans.57.10685.

Diaz, H. F., M. P. Hoerling, and J. K. Eischeid, 2001: ENSO variability, teleconnections and climate change. Int. J. Climatol., 21, 1845-1862, https://doi.org/10.1002/joc.631.

Hannachi, A., I. T. Jolliffe, and D. B. Stephenson, 2007: Empirical orthogonal functions and related techniques in atmospheric science: A review. Int. J. Climatol., 27, 1119-1152, https://doi.org/10.1002/joc.1499.

Kleiber, W., R. W. Katz, and B. Rajagopalan, 2012: Daily spatiotemporal precipitation simulation using latent and transformed Gaussian processes. Water Resour. Res., 48, W01523, https://doi.org/10.1029/2011WR011105.

,$- \ldots$, and -2013 : Daily minimum and maximum temperature simulation over complex terrain. Ann. Appl. Stat., 7, 588-612, https://doi.org/10.1214/12-AOAS602.

Lareau, N. P., E. Crosman, C. D. Whiteman, J. D. Horel, S. W. Hoch, W. O. J. Brown, and T. W. Horst, 2013: The Persistent Cold-Air Pool Study. Bull. Amer. Meteor. Soc., 94, 51-63, https://doi.org/10.1175/BAMS-D-11-00255.1.

Maurer, E. P., L. Brekke, T. Pruitt, and P. B. Duffy, 2007: Fineresolution climate projections enhance regional climate change impact studies. Eos, Trans. Amer. Geophys. Union, $\mathbf{8 8}$ 504-504, https://doi.org/10.1029/2007EO470006.

McCabe, G. J., and M. D. Dettinger, 2002: Primary modes and predictability of year-to-year snowpack variations in the western United States from teleconnections with Pacific Ocean climate. J. Hydrometeor., 3, 13-25, https://doi.org/ 10.1175/1525-7541(2002)003<0013:PMAPOY>2.0.CO;2.

Menne, M. J., I. Durre, R. S. Vose, B. E. Gleason, and T. G. Houston, 2012: An overview of the Global Historical Climatology Network-Daily database. J. Atmos. Oceanic Technol., 29, 897-910, https://doi.org/10.1175/JTECH-D-11-00103.1.

Mote, P. W., 2006: Climate-driven variability and trends in mountain snowpack in western North America. J. Climate, 19, 6209-6220, https://doi.org/10.1175/JCLI3971.1. 
Rencher, A. C., and W. F. Christensen, 2012: Methods of Multivariate Analysis. 3d ed. John Wiley and Sons, 800 pp.

Richardson, C. W., 1981: Stochastic simulation of daily precipitation, temperature, and solar radiation. Water Resour. Res., 17, 182-190, https://doi.org/10.1029/WR017i001p00182.

Smith, K., C. Strong, and S.-Y. Wang, 2015: Connectivity between historical Great Basin precipitation and Pacific Ocean variability: A CMIP5 model evaluation. J. Climate, 28, 6096-6112, https://doi.org/10.1175/JCLI-D-14-00488.1.

- _ - and F. Rassoul-Agha, 2017: A new method for generating stochastic simulations of daily air temperature for use in weather generators. J. Appl. Meteor. Climatol., 56, 953-963, https://doi.org/10.1175/JAMC-D-16-0122.1.

Stern, R. D., and R. Coe, 1984: A model fitting analysis of daily rainfall data. J. Roy. Stat. Soc., 147A, 1-34, https://doi.org/ $10.2307 / 2981736$.

Thompson, G. A., and D. B. Burke, 1974: Regional geophysics of the basin and range province. Annu. Rev. Earth Planet. Sci., 2, 213-238, https://doi.org/10.1146/annurev.ea.02.050174.001241.

Todorovic, P., and D. A. Woolhiser, 1975: A stochastic model of $\omega$-day precipitation. J. Appl. Meteor., 14, 17-24, https://doi.org/ 10.1175/1520-0450(1975)014<0017:ASMODP>2.0.CO;2.

Verdin, A., B. Rajagopalan, W. Kleiber, and R. W. Katz, 2015: Coupled stochastic weather generation using spatial and generalized linear models. Stochastic Environ. Res. Risk Assess., 29, 347-356, https://doi.org/10.1007/s00477-014-0911-6.
Wilks, D. S., 1998: Multisite generalization of a daily stochastic precipitation generation model. J. Hydrol., 210, 178-191, https://doi.org/10.1016/S0022-1694(98)00186-3.

1999a: Interannual variability and extreme-value characteristics of several stochastic daily precipitation models. Agric. For. Meteor., 93, 153-169, https://doi.org/10.1016/S0168-1923 (98)00125-7.

1999b: Simultaneous stochastic simulation of daily precipitation, temperature and solar radiation at multiple sites in complex terrain. Agric. For. Meteor., 96, 85-101, https://doi.org/ 10.1016/S0168-1923(99)00037-4.

- 2008: High-resolution spatial interpolation of weather generator parameters using local weighted regressions. Agric. For. Meteor., 148, 111-120, https://doi.org/10.1016/ j.agrformet.2007.09.005.

- 2009: A gridded multisite weather generator and synchronization to observed weather data. Water Resour. Res., 45, W10419, https://doi.org/10.1029/2009WR007902.

Wise, E. K., 2010: Spatiotemporal variability of the precipitation dipole transition zone in the western United States. Geophys. Res. Lett., 37, L07706, https://doi.org/ 10.1029/2009GL042193.

Woolhiser, D. A., 2008: Combined effects of the Southern Oscillation index and the Pacific decadal oscillation on a stochastic daily precipitation model. J. Climate, 21, 1139-1152, https:// doi.org/10.1175/2007JCLI1862.1. 